\title{
Clinical efficacy and safety of T-DMI for patients with HER2-positive breast cancer
}

This article was published in the following Dove Press journal:

OncoTargets and Therapy

29 February 2016

Number of times this article has been viewed

Bo $M a^{\prime}$

Qianqian $\mathrm{Ma}^{2}$

Hongqiang Wang ${ }^{3}$

Guolei Zhang'

Huiying Zhang'

Xiaohong Wang'

'Affiliated Central Hospital of Huzhou Teachers College, Huzhou, Zhejiang, People's Republic of China; ${ }^{2}$ University Hospital of Tuebingen, Tuebingen, Germany; ${ }^{3}$ Department of Oncology, Hospital of Zhoushan, Zhoushan, Zhejiang, People's Republic of China
Correspondence: Xiaohong Wang Affiliated Central Hospital of Huzhou Teachers College, No 198, Hongqi Road, Huzhou 313000, Zhejiang, People's Republic of China

Tel +86572202 330I

Fax +865722023301

Email dr_wangxiaohong@aliyun.com
Purpose: The aim of this study was to evaluate the therapeutic efficacy and safety of trastuzumab emtansine (T-DM1) for the treatment of patients with human epidermal growth factor receptor 2-positive breast cancer.

Methods: We performed a systemic review and meta-analysis of the relevant published clinical studies. A computerized search was performed for controlled clinical trials of T-DM1 in targeted treatment. Overall survival, progression-free survival, objective response rate, symptom progression free, and adverse events (AEs) were evaluated.

Results: Eight eligible trials with a total of 2,016 patients with breast cancer were included in the present meta-analysis. The treatment of patients with breast cancer with T-DM1 was associated with significantly increased overall and progression-free survival when compared with controls $(P<0.0001)$. An analysis of the objective response rate and symptom progression free also demonstrated favorable results for T-DM1 treatment $(P \leq 0.0001)$. There was no significant difference between the T-DM1 and control groups with respect to nonhematologic or hematologic AEs ( $P=0.99$ and $P=0.30$, respectively).

Conclusion: Overall, T-DM1 is efficacious in the treatment of patients with human epidermal growth factor receptor 2-positive breast cancer and low rates of AEs compared with controls.

Keywords: breast cancer, meta-analysis, HER2, T-DM1, efficacy

\section{Introduction}

Breast cancer accounts for $\sim 28 \%$ of all new cancers in women. It is a major health problem and the second leading cause of cancer death in the USA. ${ }^{1,2}$ Breast cancer is now known to be a heterogeneous disease, which is characterized by a variety of biological drivers and related clinical results. ${ }^{3}$ Human epidermal growth factor receptor 2 (HER2) is a member of the epidermal growth factor receptor family of transmembrane receptors. ${ }^{4-6}$ The other three family members are HER1 (also known as epidermal growth factor receptor), HER3 (ErbB3), and HER4 (ErbB4). Protein overexpression and/or gene amplification of HER2 (also known as ErbB2, $p 185^{\text {HER2 }}$, and neu) is present in $\sim 15 \%-25 \%$ of new patients with breast cancer. ${ }^{4,7,8}$ HER2 overexpression results in an aggressive form of breast cancer that is associated with poor clinical outcomes and greater therapeutic resistance compared with HER2-normal patients..$^{9,10}$ The clinical outcomes of these patients with breast cancer have greatly improved with the development of HER2-targeted therapies, but therapeutic resistance is still common, and the management of toxicity remains a challenge. ${ }^{11}$ Therefore, new treatments are needed for patients with breast cancer who demonstrate disease progression following HER2-targeted therapies.

HER2-targeted therapies include trastuzumab, the first humanized monoclonal antibody, and newer drugs such as lapatinib, ertumaxomab, and pertuzumab. Trastuzumab 
(Herceptin; Genentech, Inc., South San Francisco, CA, USA) combined with others is the standard treatment for HER2positive patients with early or metastatic breast cancer. ${ }^{12}$ The molecular mechanisms behind trastuzumab include mitogenactivated protein kinases and phosphoinositide 3 kinase (PI3K)/AKT signaling inhibition, antibody-dependent cellmediated cytotoxicity, induction of apoptosis, and prevention of HER2 ectodomain cleavage. ${ }^{13,14} \mathrm{DM} 1$, an antimicrotubule agent, is derived from maitansine. Trastuzumab emtansine (T-DM1, Kadcyla), which has been developed by Genentech, Inc. and ImmunoGen, Inc. (Waltham, USA), combines trastuzumab and DM1, allowing preferential intracellular drug delivery to HER2-positive tumor cells. , $^{2,15,16}$

T-DM1, an antibody-drug conjugate, was granted marketing approval by the US Food and Drug Administration in 2013. Because the toxicity associated with chemotherapy is a large source of distress for patients, the antibody-drug conjugate is considered to be a promising treatment. ${ }^{7,17}$ T-DM1 combines the antitumor effects of trastuzumab with a cytotoxic antimicrotubule agent (DM1) that is released within HER-positive tumor cells. In August 2015, we identified 48 registered Phase I, II, or III clinical trials on the treatment of breast cancer with T-DM1 on ClinicalTrials.gov website, using the keywords "T-DM1" and "breast cancer". Ten of those clinical trials have been completed.

In the current study, we performed a systematic review and meta-analysis of clinical trials to assess the efficacy and safety of T-DM1 in the treatment of patients with breast cancer. The aim was to evaluate the clinical response to T-DM1 by assessing overall survival (OS), progression-free survival (PFS), the objective response rate (ORR), and symptom progression free (SPF), in addition to adverse events (AEs).

\section{Materials and methods}

\section{Search strategy, study design, and eligibility criteria}

PubMed, ScienceDirect, the Cochrane Central Register of Controlled Trials, the China Science and Technology Journal Database, the Wanfang Database, and China Journal Net were searched for relevant studies published from 1980 until August 5, 2015. The search strategy included the keywords ("trastuzumab emtansine" or "T-DM1" or "trastuzumab-DM1") and "HER2" and ("breast cancer" or "metastatic breast cancer") and clinical trial, without language or time limitations. We also searched the ClinicalTrials.gov website for information on ongoing trials, using the keywords ("trastuzumab emtansine" or "T-DM1" or "trastuzumab-DM1") and "breast cancer". Publication citations displayed at the bottom of the "Full Text View" tab of a study record under the "More Information" heading. In addition, relevant review papers, postgraduate articles, and previously published trials were examined to identify further relevant trials. We carefully searched the latest reports of the European Cancer Conference and the American Society of Clinical Oncology Annual Meeting and the Word Conference on Breast Cancer. Studies were eligible for inclusion if they 1) were English or Chinese studies on T-DM1 treatment of patients with breast cancer; 2) included an appropriate control arm; and 3) enrolled ten or more patients. Reviews and Phase I studies were excluded. In addition, studies on cell lines and animals, case reports, studies investigating multiple types of cancer, and those lacking patients' details were excluded.

\section{Data selection criteria and quality assessment}

Data extraction and study selection were independently conducted by two reviewers (Qianqian Ma and Hongqiang Wang) using a standardized approach. Any differences were adjudicated by a third reviewer (Bo Ma), based on the original publication. Study features extracted included the first author's name, country and year of publication, clinical trial phase, tumor characteristics, number of patients, sample size per arm, mean patient age, previous treatments, T-DM1 dose and route of administration, and number of estrogen receptor-, and/or progesterone receptor-positive patients. Any data that could not be directly obtained from the articles were calculated from the graphed data using Adobe Photoshop and Illustrator.

\section{Definition of outcome measures}

OS was defined as the time from study treatment initiation to death from any cause. PFS was defined as the interval from study treatment initiation to the first occurrence of progressive disease. ORR was defined as a complete or partial response on two consecutive tumor assessments not $<4$ weeks apart. SPF was defined as the time from study treatment initiation to symptom progression. The primary endpoints were OS and PFS. Secondary outcomes were ORR, SPF, and safety. AEs and toxicities were graded according to the National Cancer Institute Common Toxicity Criteria.

\section{Statistical analysis}

Data on OS, PFS, ORR, SPF, and AEs were extracted from the identified trials. In this analysis, we compared the T-DM1 treatment groups form the trials with their respective control 
groups. The meta-analysis was performed using Review Manager Version 5.0 (Nordic Cochrane Centre) and Stata Version 12.0 (StataCorp LP, College Station, TX, USA). The effects of T-DM1 treatment were reflected by odds ratios (ORs). Fixed- and random-effects models were used to estimate the effects of T-DM1 treatment. Heterogeneity among the trials was assessed to determine which model should be used. We used the $\chi^{2}$-based $Q$-test to examine heterogeneity among the studies, and the significance level was fixed at $P<0.10$. The quantity $I^{2}$ was also calculated to evaluate heterogeneity, and $I^{2}>50 \%$ was considered to indicate a high level of heterogeneity. A random-effects model was used when statistical heterogeneity was confirmed; otherwise, a fixed-effects model was employed. $P \leq 0.05$ was considered to be statistically significant, and all reported $P$-values resulted from two-sided version tests of the respective tests. ${ }^{18}$ To assess the possibility of publication bias, Egger's test and Begg's test were used.

\section{Results}

\section{Trial selection}

The data search yielded 119 references, 75 of whom were excluded for various reasons (Figure 1). A further 36 studies were excluded because they were published in other languages, did not provide detailed enough clinical data, or did not have an appropriate control arm. Finally, eight articles reporting clinical trials of T-DM1-based therapy for breast cancer were selected for this meta-analysis (Figure 1). ${ }^{19-26}$

\section{Baseline patient characteristics}

The baseline characteristics of the patients in the eight selected publications that described six clinical trials are listed in Table 1. The trials involved a total of 2,016 patients with locally advanced or metastatic breast cancer. All the eight papers were fully published and described four Phase II trials ${ }^{19,21,22,24,25}$ and two Phase III trials. ${ }^{20,23,26}$ The patients enrolled were between 24 years and 84 years of age, with a median age of $>52$ years. Patients with breast cancer had received previous treatments, including trastuzumab, anthracyclines, taxanes, capecitabine, carboplatin, lapatinib, endocrine therapy, and radiotherapy. In all the trials, patients assigned to T-DM1 were given a dose of $3.6 \mathrm{mg} / \mathrm{kg}$ intravenously every 3 weeks, while patients assigned to the control arms received trastuzumab, docetaxel, lapatinib, capecitabine, or hormonal therapy. A total of 1,065 patients (53\%) were estrogen receptor and/or progesterone receptor positive.

\section{Overall survival}

\section{Two-month, 4-month, and 6-month OS}

Information on the 2-month, 4-month, and 6-month OS rates was available from two trials..$^{20,26}$ These two trials contained a total of 1,593 patients, of whom 899 patients received T-DM1, and 694 controls did not receive T-DM1

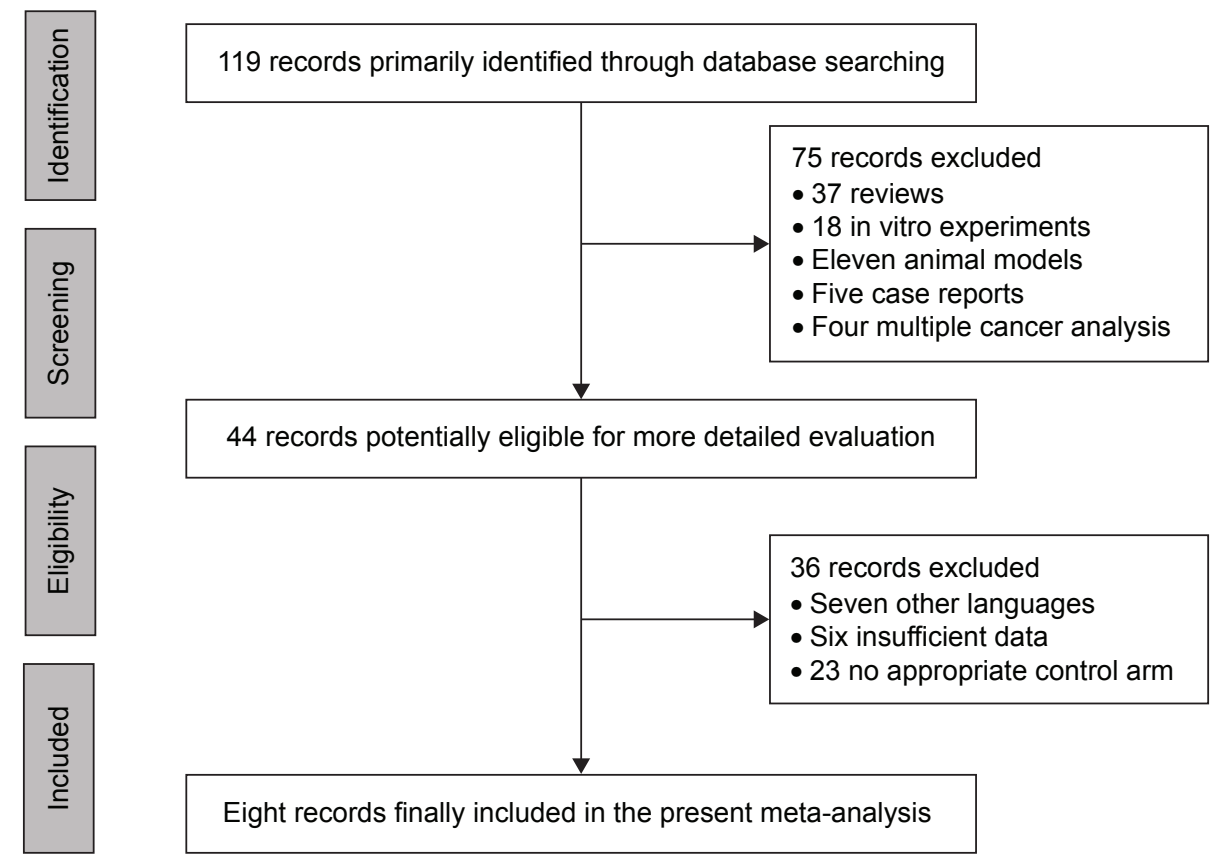

Figure I Flow diagram of the study identification, screening, and inclusion process. 


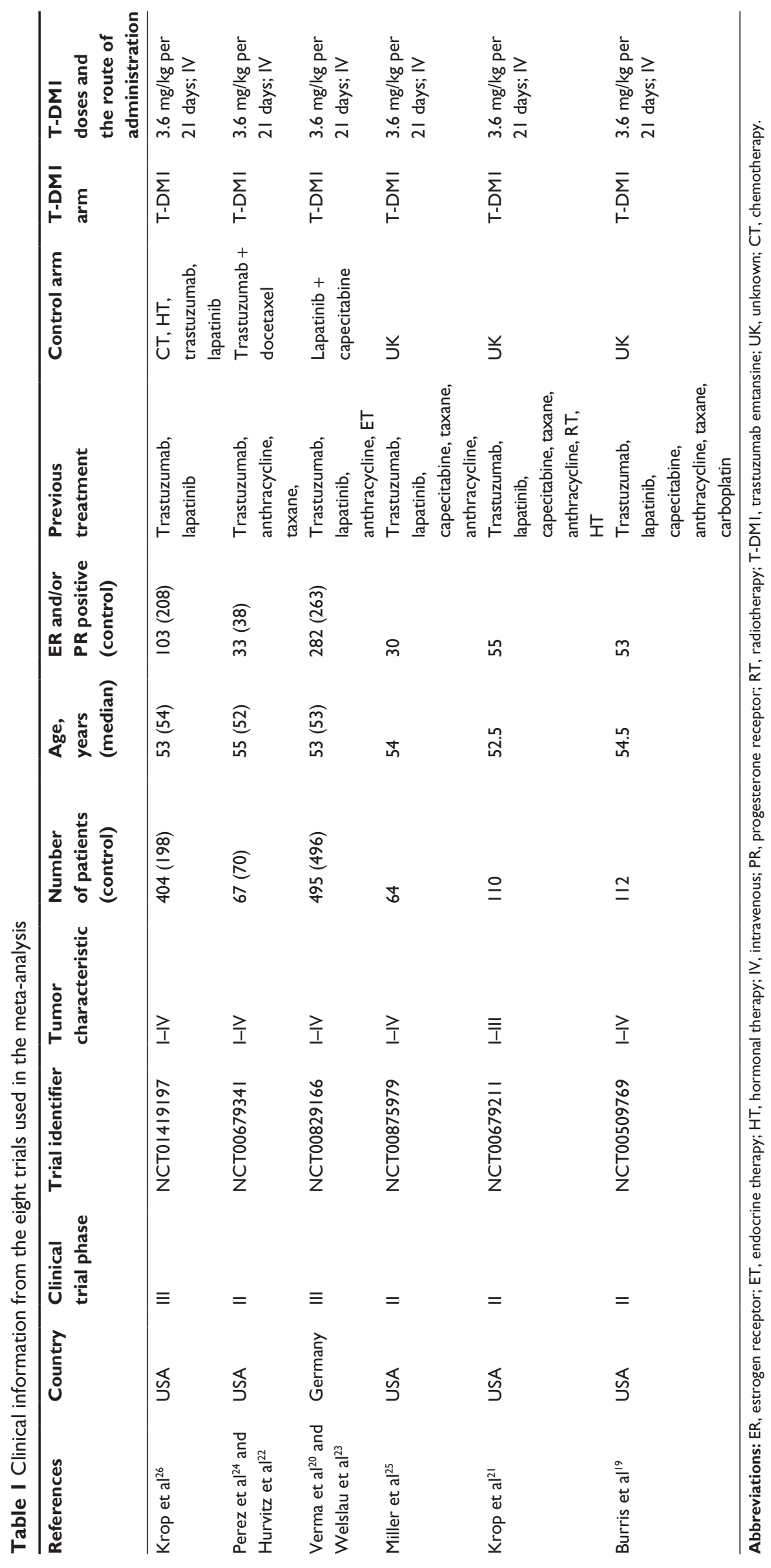


or were HER2 negative. The 2-month OS rate was $96 \%$ (866/899) for patients receiving T-DM1 compared with $92 \%(640 / 694)$ for controls. The meta-analysis showed a significantly higher 2-month OS rate for patients receiving T-DM1 compared with the control group (OR, 2.73; 95\% confidence interval [CI], 1.73-4.31; $P<0.0001)$. Cochran's $Q$-test yielded a $P$-value of 0.84 , and the corresponding $I^{2}$ was $0 \%$, indicating that the degree of variability between the two trials was consistent with what would be expected by chance alone (Figure 2A). The 4-month OS rate for the 899 patients in the T-DM1 group was $88 \%$ compared with $83 \%$ for controls. Pooled analysis showed that T-DM1 significantly increased the 4-month OS rate compared with the control group (OR, 2.11; 95\% CI, 1.55-2.87; $P<0.0001)$. Cochran's $Q$-test yielded a $P$-value of 0.95 , and the corresponding $I^{2}$ was $0 \%$ (Figure 2A). A significant difference was also demonstrated in the 6-month OS rate (OR, 1.60; 95\% CI, 1.23-2.10; $P=0.0006)$. Cochran's $Q$-test yielded a $P$-value of 0.76 , and the corresponding $I^{2}$ was $0 \%$ (Figure $2 \mathrm{~A}$ ).

\section{Eight-month, 10-month, and 12-month OS}

Information on the 8-month, 10-month, and 12-month OS rates was available from two trials, ${ }^{20,26}$ including a total of 1,593 patients ( 899 of whom received T-DM1; Figure 2B). T-DM1 treatment was associated with 8-month, 10-month, and 12 -month OS rates of $63 \%, 54 \%$, and $42 \%(566 / 899$, $483 / 899$, and $379 / 899$ patients), respectively. Both the trials showed a longer OS for patients who received T-DM1 in comparison with controls or HER2-negative patients at 8 months, 10 months, and 12 months. The estimated pooled OR showed significantly improved 8-month and 12-month OS rates for patients with breast cancer receiving T-DM1 (OR, 1.56; 95\% CI, 1.15-2.12; $P=0.005$ and OR, 1.61; 95\% CI, 1.25-2.07; $P=0.0002$, respectively), but the 10-month OS rate was not significantly improved (OR, 1.46; 95\% CI, 0.84-2.54; $P=0.17)$. The overall Cochran's $Q$-test had a $P$-value of 0.39 , and the corresponding $I^{2}$ was $5 \%$.

\section{Fourteen-month and 16-month OS}

Information on the 14-month and 16-month OS rates was available from two trials, ${ }^{20,26}$ which contained a total of 1,593 patients ( 899 of whom received T-DM1; Figure 2C). T-DM1 was associated with 14-month and 16-month OS rates of $33 \%$ and $27 \%$ (300/899 and 242/899 patients), respectively. Both the trials showed a longer OS for patients receiving T-DM1 in comparison with controls or HER2-negative patients at 14 months. The estimated pooled OR showed a highly improved 14-month and 16-month OS rate for patients who received T-DM1 (OR, $1.53 ; 95 \%$ CI, $1.20-1.96 ; P=0.0007$ and OR, $1.37 ; 95 \%$ CI, 1.07-1.76; $P=0.01$, respectively). The overall Cochran's $Q$-test yielded a $P$-value of 0.75 , and the corresponding $I^{2}$ was $0 \%$.

\section{Progression-free survival}

\section{Two-month, 4-month, and 6-month PFS}

Information on the 2-month, 4-month, and 6-month PFS rates was available from six trials. ${ }^{19-22,25,26}$ These six trials included a total of 1,984 patients, 1,141 of whom received T-DM1 and 843 controls who did not receive T-DM1 treatment or who were HER2 negative. The 2-month PFS rate was $83 \%(952 / 1,141)$ for patients receiving T-DM1 and $76 \%$ (641/843) for controls. The meta-analysis showed a significantly higher 2-month PFS rate for patients receiving T-DM1 compared with controls (OR, 1.93; 95\% CI, 1.07-3.45; $P=0.03$; Figure 3A). Cochran's $Q$-test yielded a $P$-value of 0.001 , and the corresponding $I^{2}$ was $75 \%$. The 4-month PFS rate for the 1,141 patients in the T-DM1 group was $65 \%$ compared with $56 \%$ for 843 controls. The pooled analysis showed that T-DM1 treatment did not significantly increase the 4-month PFS rate compared with controls (OR, 1.89; 95\% CI, 0.97-3.68; $P=0.06$; Figure 3A). Cochran's $Q$-test yielded $P<0.00001$, and the corresponding $I^{2}$ was $85 \%$. The 6-month PFS rate for the 1,141 patients in the T-DM1 group was $42 \%$ compared with $33 \%$ for 843 controls. A significant difference was also demonstrated in the 6-month PFS rate (OR, 1.90; 95\% CI, 1.13-3.19; $P=0.02$ ). Cochran's $Q$-test yielded a $P$-value of 0.01 , and the corresponding $I^{2}$ was $67 \%$ (Figure 3A).

\section{Eight-month, 10-month, and 12-month PFS}

Information on the 8-month, 10-month, and 12-month PFS rates was available from six trials, ${ }^{19-22,25,26}$ which included a total of 1,984 patients (1,141 of whom received T-DM1; Figure 3B). T-DM1 treatment was associated with 8-month, 10 -month, and 12-month PFS rates of $31 \%, 21 \%$, and $14 \%$ $(355 / 1,141,240 / 1,141$, and $165 / 1,141$ patients), respectively, compared with only $24 \%, 14 \%$, and $9 \%$ (199/843, 116/843, and $75 / 843$ patients), respectively, in the control group. The results showed a longer PFS for patients who received T-DM1 in comparison with controls or HER2-negative patients at 8 months, 10 months, and 12 months. The estimated pooled OR showed a significantly improved 8-month,10-month, and 12-month PFS rates for patients with breast cancer receiving T-DM1 (OR, 2.00; 95\% CI, 1.69-2.37; $P<0.00001)$. 


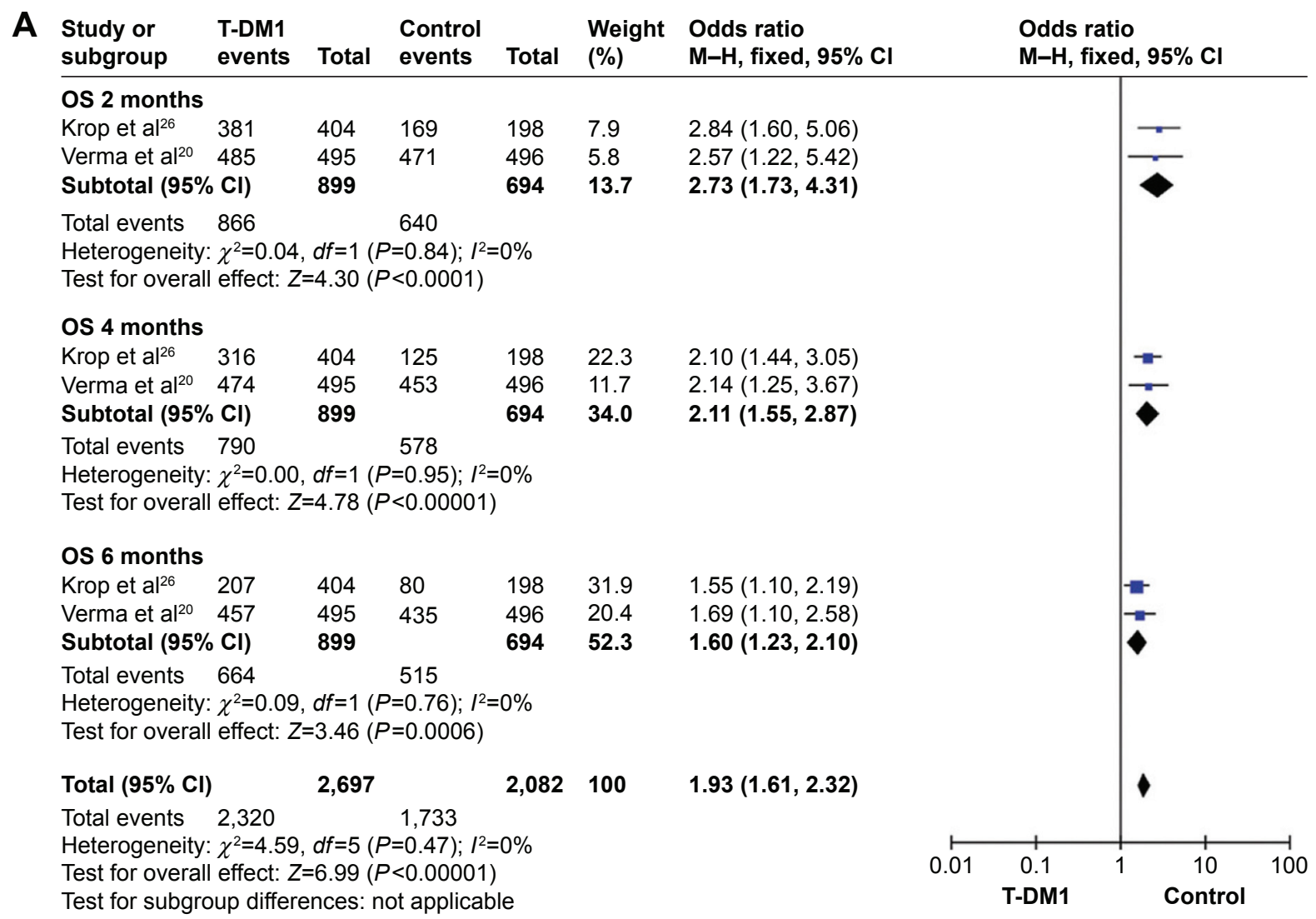

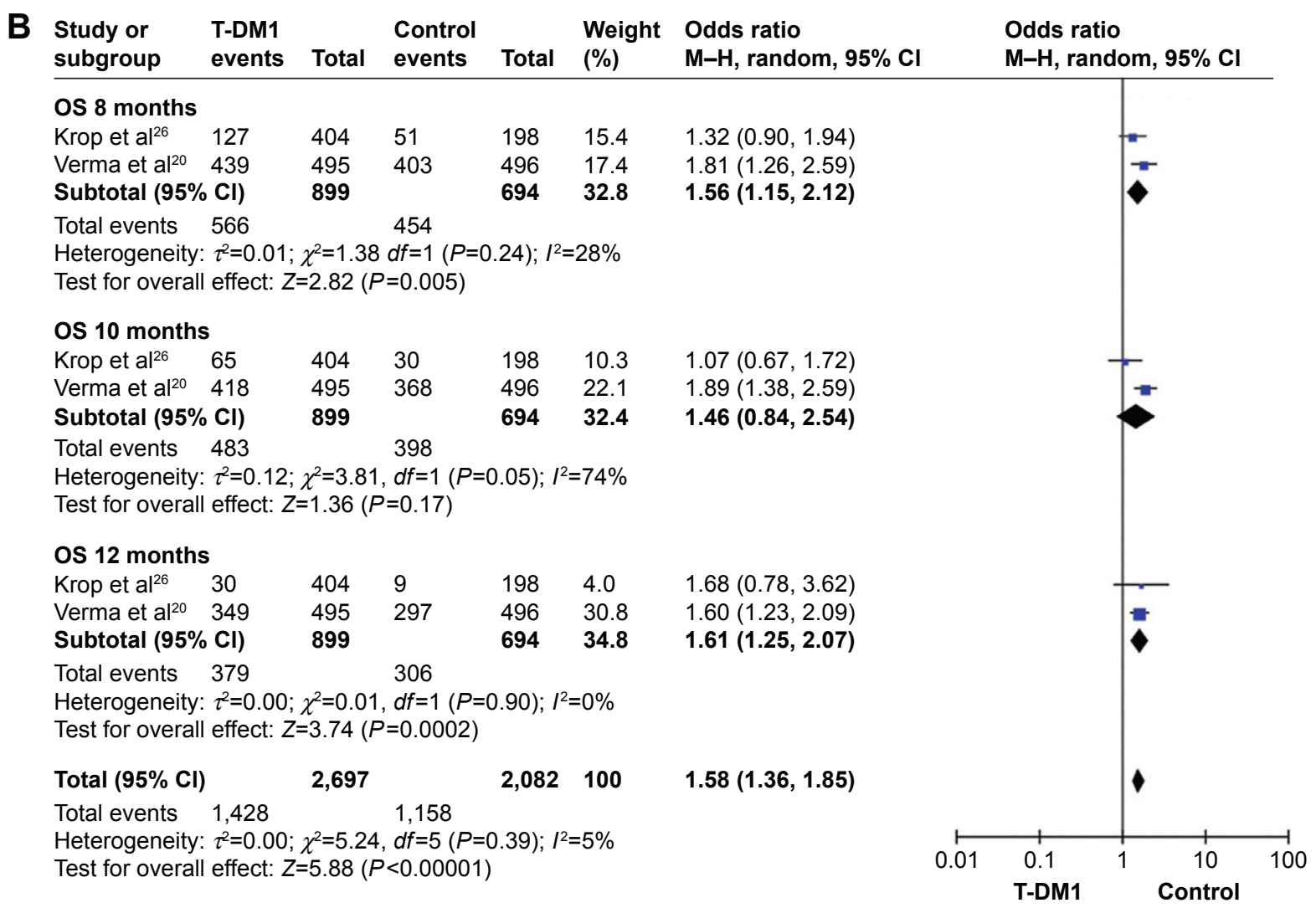

Figure 2 (Continued) 


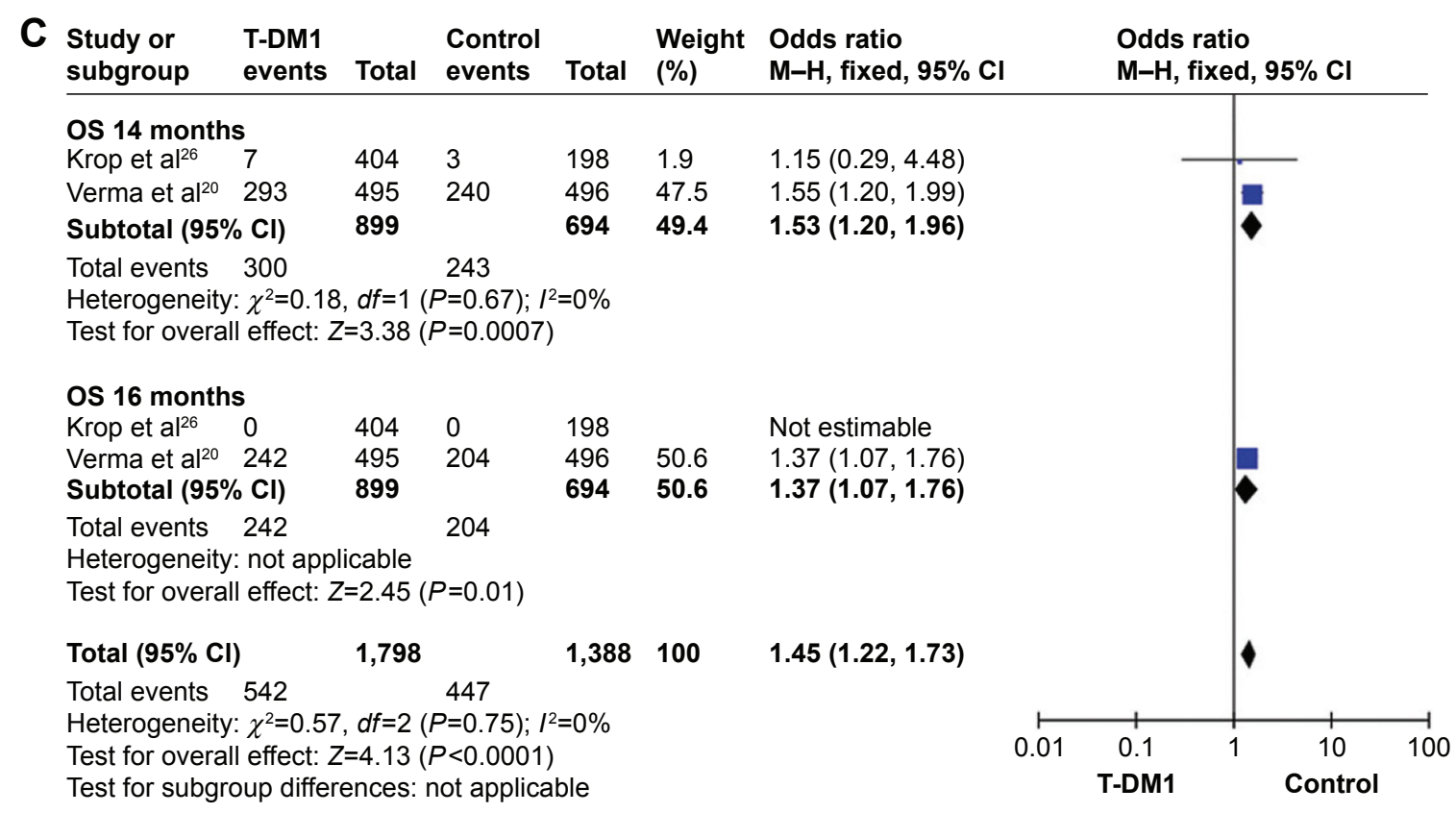

Figure 2 Forest plots of OS rates between patients undergoing T-DMI therapy and controls at (A) 2 months, 4 months, and 6 months, (B) 8 months, 10 months, and 12 months, and (C) 14 months and 16 months.

Notes: Fixed- and random-effects models ( $\mathrm{M}-\mathrm{H}$ method) were used. Each trial is represented by a square, the center of which gives the odds ratio for that trial. The size of the square is proportional to the information in that trial. The ends of the horizontal bars denote $95 \% \mathrm{Cls}$. Black diamonds give the overall odds ratios for the combined results of all trials. Abbreviations: Cls, confidence intervals; $d f$, degrees of freedom; $\mathrm{M}-\mathrm{H}$, Mantel-Haenszel; OS, overall survival; T-DMI, trastuzumab emtansine.

The overall Cochran's $Q$-test had a $P$-value of 0.37 , and the corresponding $I^{2}$ was $7 \%$.

Fourteen-month, 16-month, and I8-month PFS Information on the 14-month PFS rate was available from six trials, ${ }^{19-22,25,26}$ which included a total of 1,984 patients $(1,141$ of whom received T-DM1; Figure 3C). T-DM1 treatment was associated with a 14-month PFS rate of 9\% (107/1,141 patients) compared with $6 \%(47 / 843$ patients $)$ in the control group. The estimated pooled OR showed a significantly improved 14-month PFS rate for patients who received T-DM1 (OR, 2.52; 95\% CI, 1.73-3.65; P<0.0001). Cochran's $Q$-test yielded a $P$-value of 0.60 , and the corresponding $I^{2}$ was $0 \%$. Information on the 16-month and 18-month PFS rates was available from four trials, ${ }^{20-22,25}$ which included a total of 1,287 patients (663 of whom received T-DM1). T-DM1 treatment was associated with 16-month and 18-month PFS rates of $11 \%$ and $8 \%(70 / 663$ and 50/663 patients, respectively, compared with $5 \%$ and $3 \%$ (33/624 and 20/624 patients), respectively, in the control group. The results showed a higher PFS rate for patients with breast cancer receiving T-DM1 in comparison with controls at 16 months and 18 months. The estimated pooled OR showed a highly improved 16-month and 18-month PFS rates for patients who received T-DM1 treatment (OR, 2.20; 95\% CI, 1.42-3.41; $P=0.0004$ and
OR, $2.59 ; 95 \%$ CI, $1.52-4.41 ; P=0.0005$, respectively). The overall Cochran's $Q$-test yielded a $P$-value of 0.66 , and the corresponding $I^{2}$ was $0 \%$.

\section{Objective response rate}

Information on the ORR was available from five trials, ${ }^{19-21,24,25}$ which included a total of 1,155 patients (627 of whom received T-DM1; Figure 4). The ORR was 44\% (278/627) for patients receiving T-DM1 compared with 33\% (175/528) in the control group. Pooled analysis indicated that T-DM1 treatment was associated with a favorable result with respect to ORR (OR, 1.82; 95\% CI, 1.41-2.34; $P<0.00001)$. Cochran's $Q$-test had a $P$-value of 0.24 , the corresponding $I^{2}$ was $28 \%(<50 \%)$, and a fixed-effects model was used (Figure 4).

\section{Symptom progression free}

\section{Two-month, 4-month, and 6-month SPF}

Information on the 2-month, 4-month, and 6-month SPF rates was available from two trials, ${ }^{20,22}$ which included a total of 1,027 patients (515 of whom received T-DM1; Figure 5A). T-DM1 treatment was associated with 2-month, 4-month, and 6-month SPF rates of $77 \%, 57 \%$, and $37 \%(395 / 515$, $296 / 515$, and $193 / 515$ patients), respectively, compared with only $67 \%, 45 \%$, and $37 \%(341 / 512,231 / 512$, and 


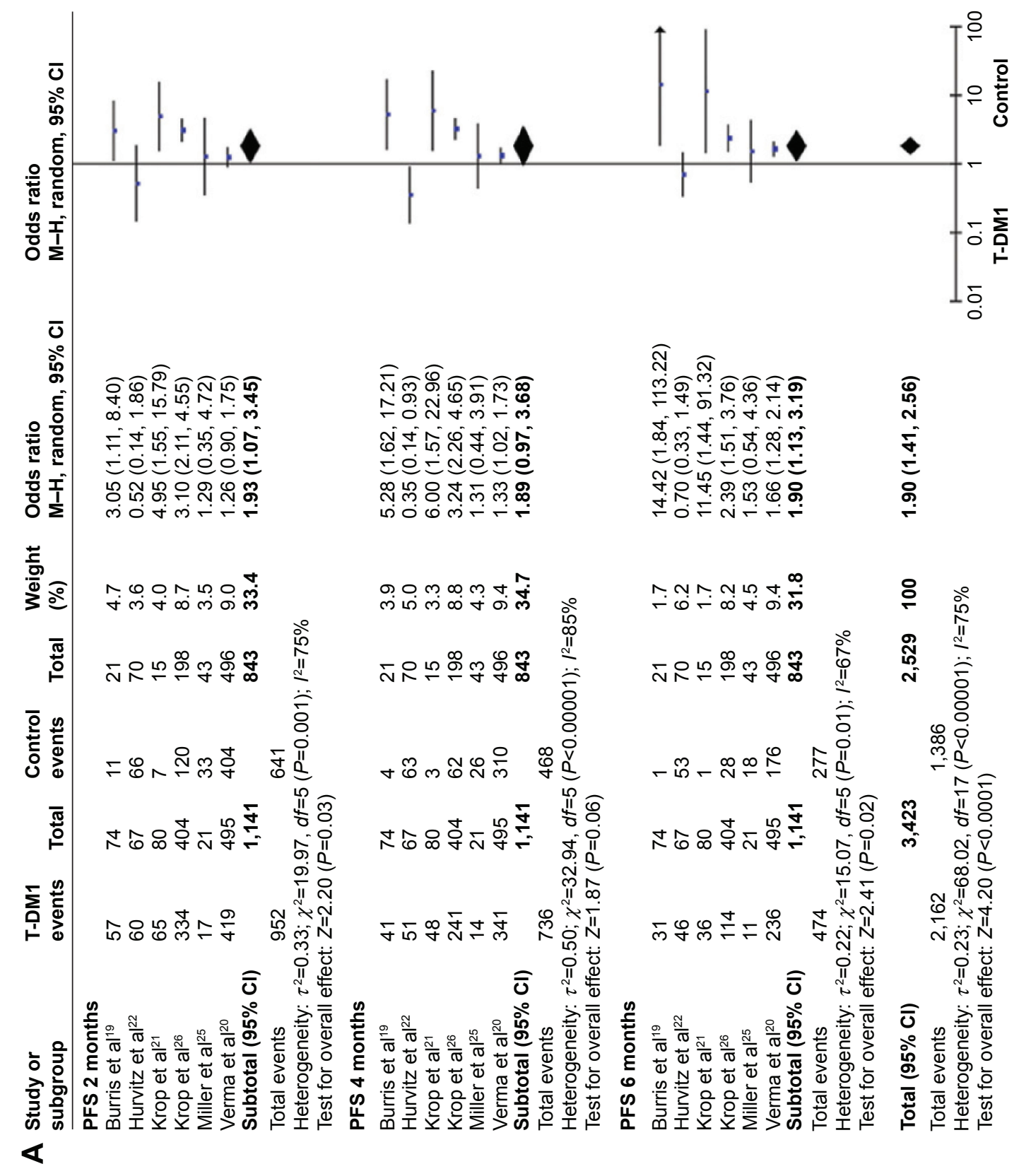




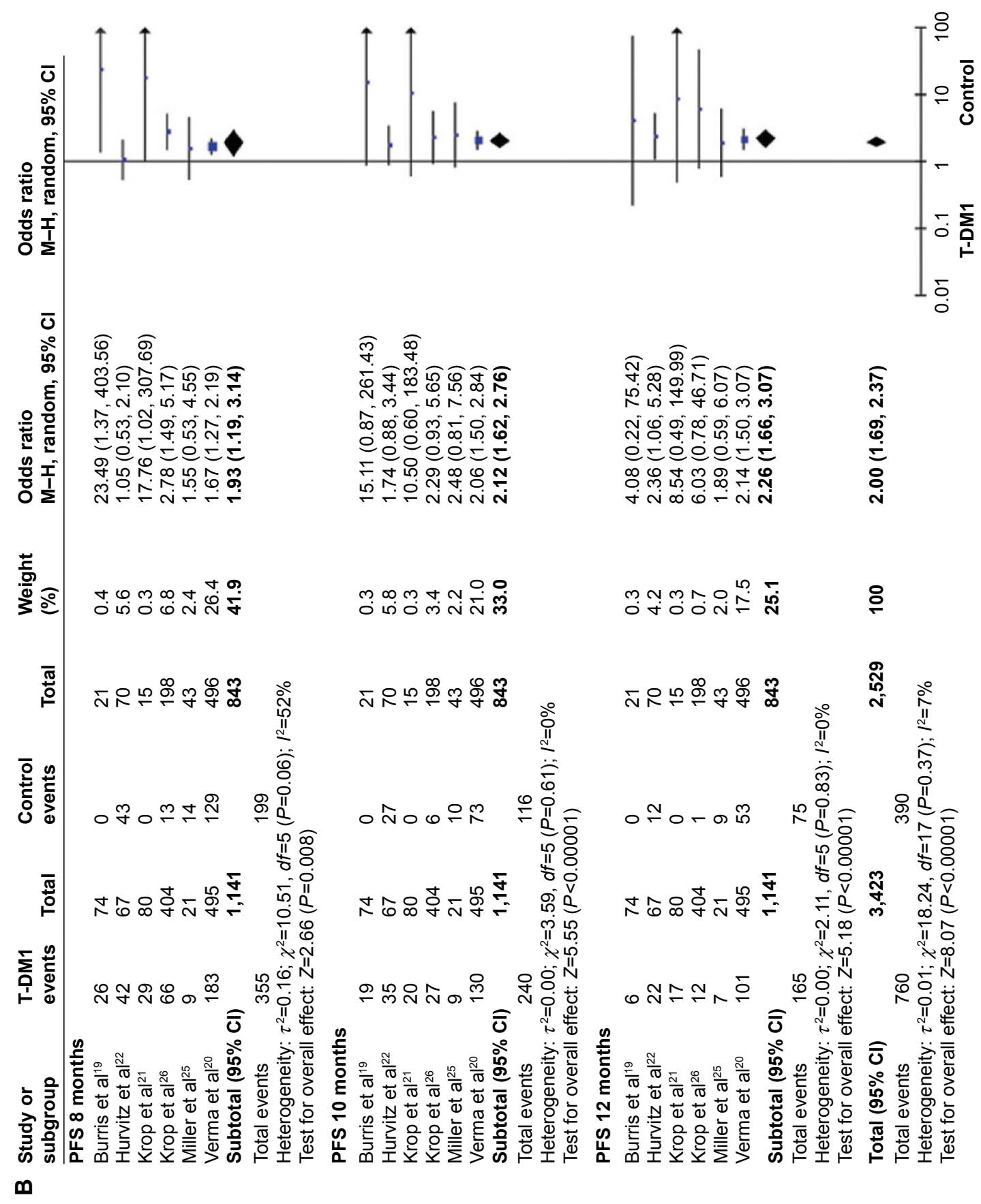




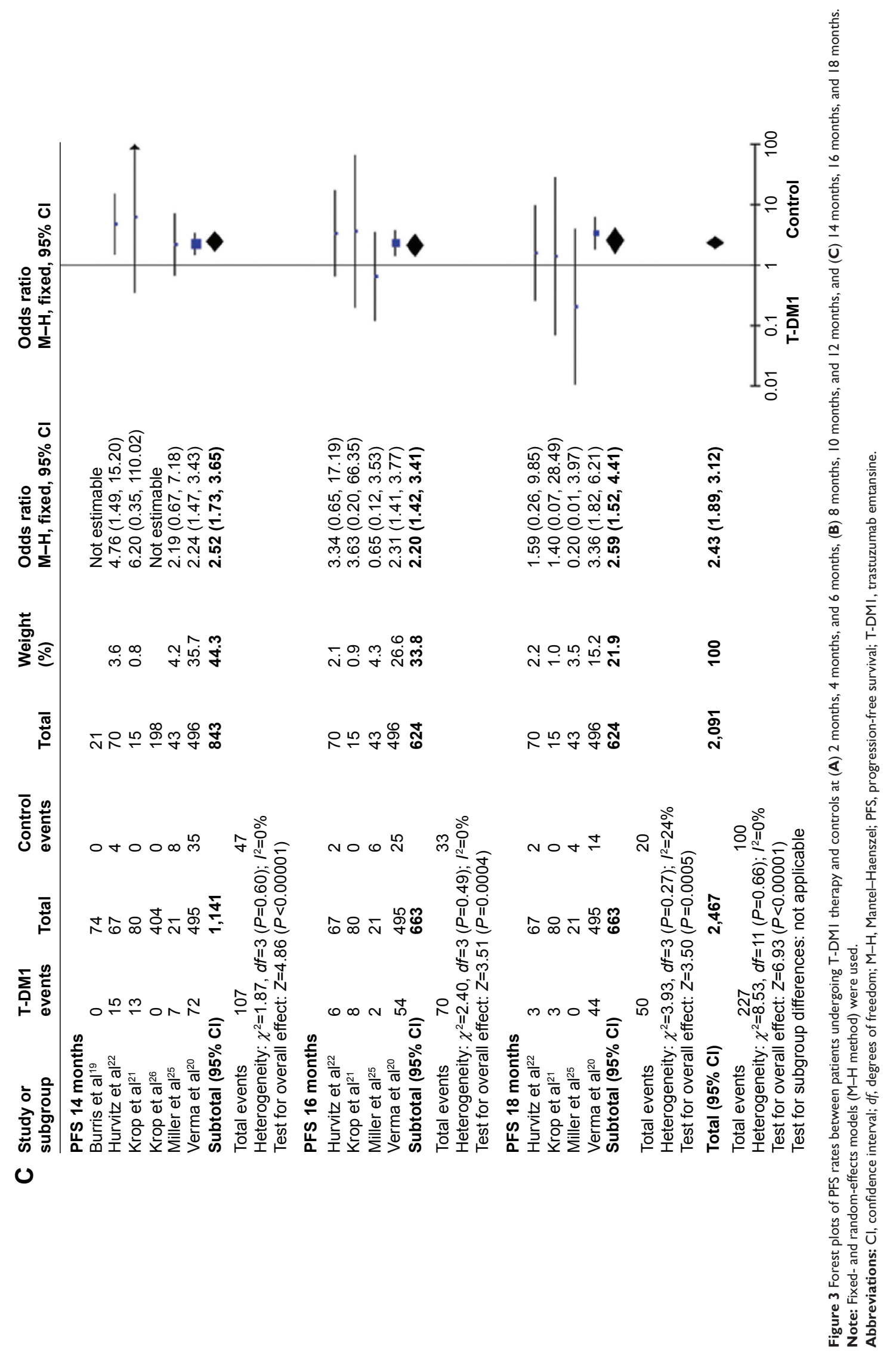




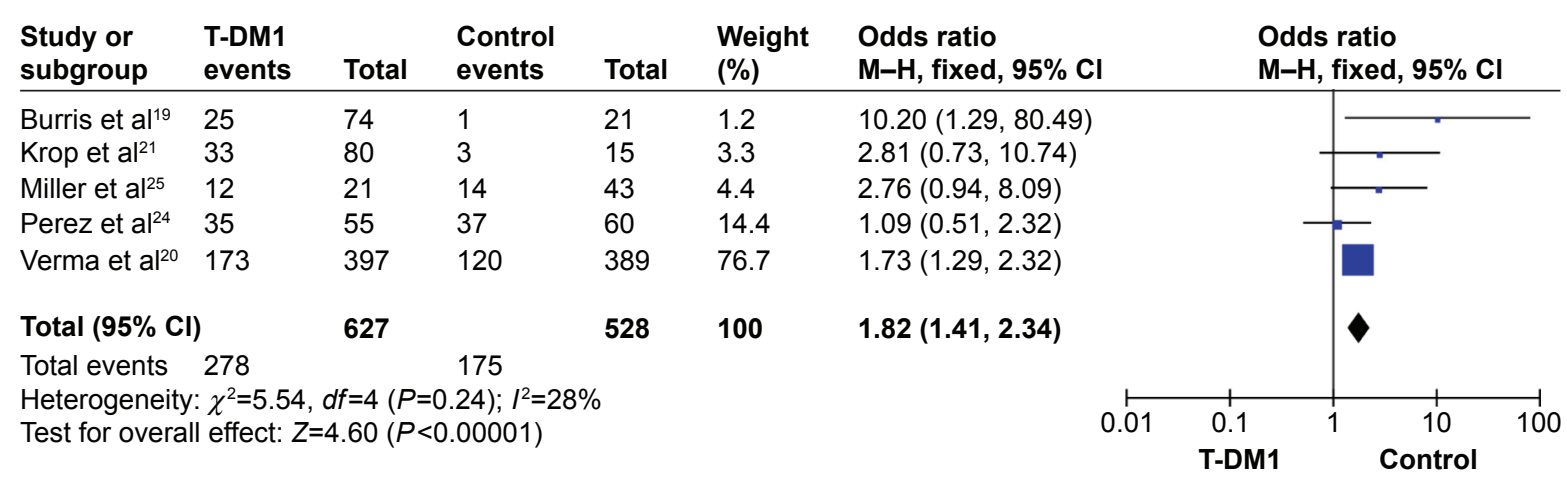

Figure 4 Comparison of the ORRs between patients undergoing T-DMI therapy and controls.

Note: The fixed-effects model ( $\mathrm{M}-\mathrm{H}$ method) was used.

Abbreviations: $\mathrm{Cl}$, confidence interval; $d f$, degrees of freedom; $\mathrm{M}-\mathrm{H}$, Mantel-Haenszel; ORRs, objective response rates; T-DMI, trastuzumab emtansine.

$140 / 512$ patients) in the control group. Both the trials showed a higher SPF rate for patients who received T-DM1 in comparison with controls at 2 months, 4 months, and 6 months. The estimated pooled OR showed significantly improved 2-month, 4-month, and 6-month SPF rates for patients with breast cancer receiving T-DM1 (OR, 2.01; 95\% CI, 0.96-4.17; $P=0.06$; OR, 1.64 ; 95\% CI, $1.28-2.10 ; P<0.0001$; and OR, 1.60; 95\% CI, 1.23-2.08; $P=0.0005$, respectively; Figure 5A). The overall Cochran's $Q$-test had a $P$-value of 0.68 , and the corresponding $I^{2}$ was $0 \%$.
Eight-month, I0-month, and I2-month SPF

Information on the 8-month, 10-month, and 12-month SPF rates was available from two trials, ${ }^{20,22}$ which included a total of 1,027 patients (515 of whom received T-DM1; Figure 5B). The 8-month, 10-month, and 12-month SPF rates in the T-DM1 arm were $29 \%, 20 \%$, and $15 \%(151 / 515$, $104 / 515$, and $76 / 515$ patients), respectively, compared with $19 \%, 12 \%$, and $10 \%(98 / 512,63 / 512$, and $49 / 512$ patients), respectively, in the control group. The results showed that the SPF rate was significantly higher in the T-DM1 arm

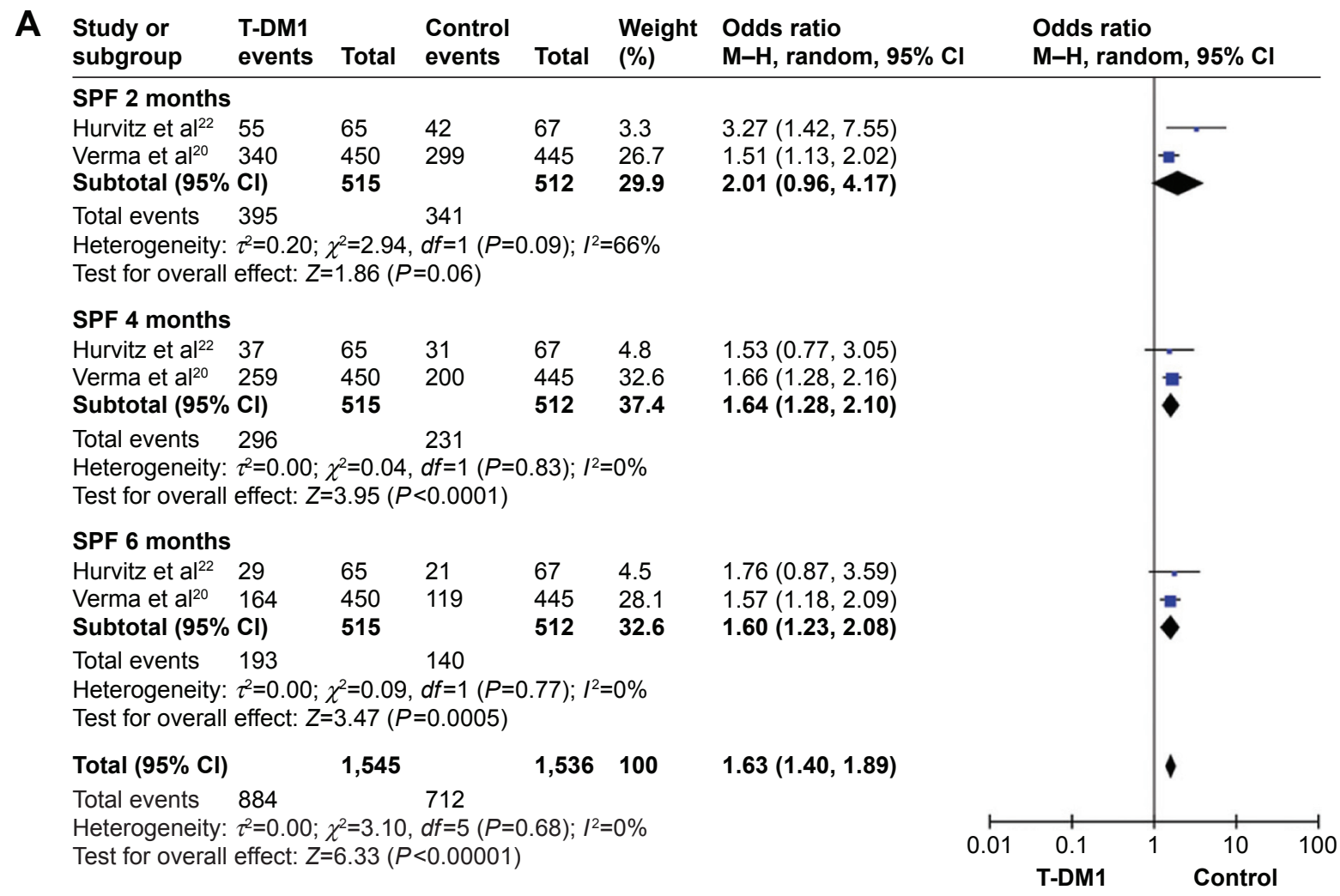

Figure 5 (Continued) 


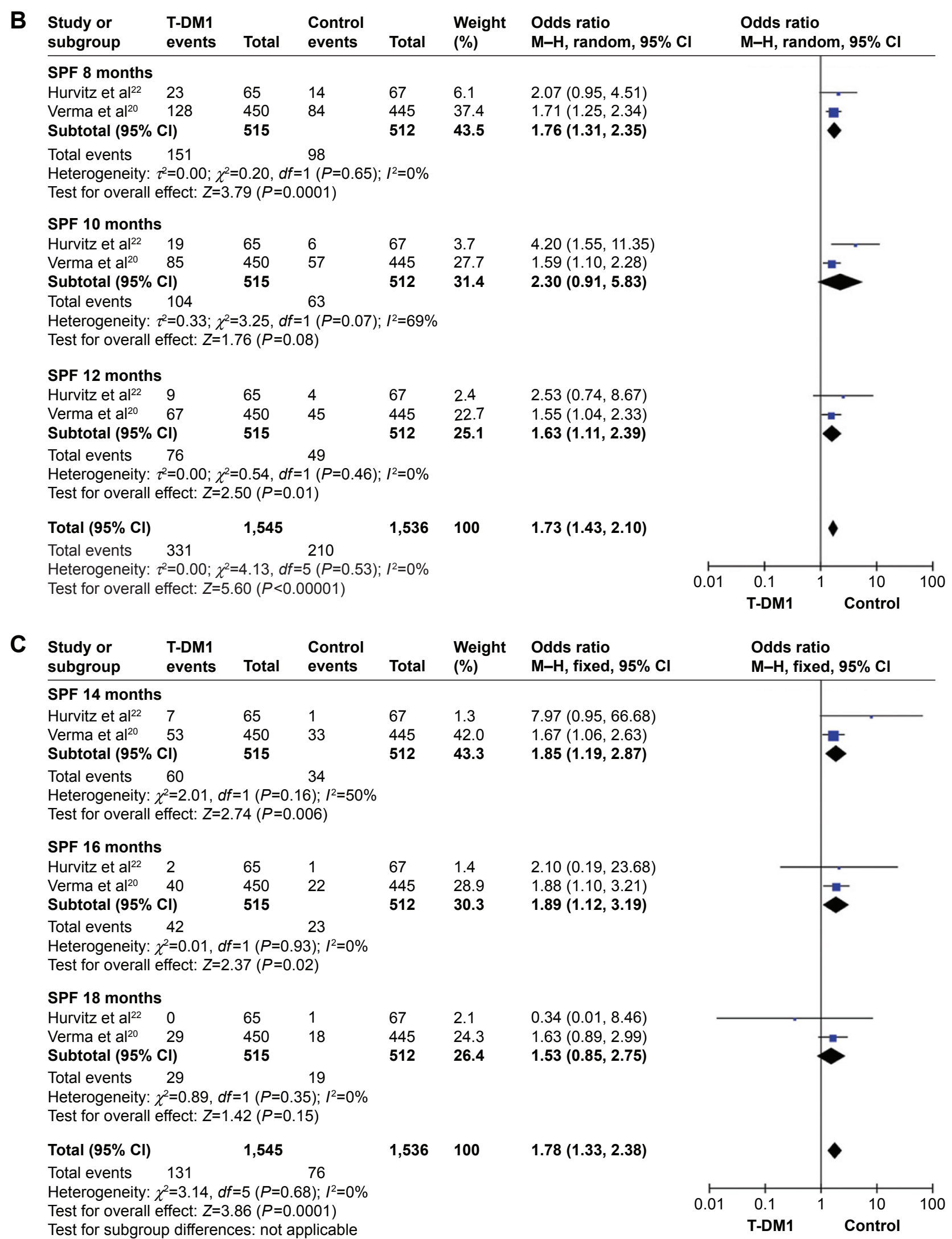

Figure 5 Forest plots of SPF between patients undergoing T-DMI therapy and controls at (A) 2 months, 4 months, and 6 months, (B) 8 months, I0 months, and I 2 months, and (C) 14 months, 16 months, and 18 months.

Note: Fixed- and random-effects meta-analysis models ( $\mathrm{M}-\mathrm{H}$ method) were used.

Abbreviations: $\mathrm{Cl}$, confidence interval; $d f$, degrees of freedom; $\mathrm{M}-\mathrm{H}$, Mantel-Haenszel; SPF, symptom progression free; T-DMI, trastuzumab emtansine. 
in comparison with controls at 8 months, 10 months, and 12 months. The meta-analysis showed a significant improvement in the 8-month, 10-month, and 12-month SPF rates in patients who received T-DM1 compared with controls (OR, 1.76; 95\% CI, 1.31-2.35; $P=0.0001$; OR, 2.30; 95\% CI, 0.91-5.83; $P=0.08$; and OR, 1.63; 95\% CI, 1.11-2.39; $P=0.01$, respectively; Figure $5 \mathrm{~B})$. The overall Cochran's $Q$-test had a $P$-value of 0.53 . The corresponding $I^{2}$ was $0 \%$, indicating that the degree of variability between the trials was consistent with what would be expected by chance alone.

\section{Fourteen-month, I6-month, and I8-month SPF}

Information on the 14-month, 16-month, and 18-month SPF rates was available from two trials. ${ }^{20,22}$ These trials included a total of 1,027 patients (515 of whom received T-DM1 treatment and 512 who acted as controls; Figure 5C). The 14-month, 16-month, and 18-month SPF rates were 12\%, 8\%, and $6 \%(60 / 515,42 / 515$, and $29 / 515$ patients $)$, respectively, for patients receiving T-DM1 compared with 7\%, 4\%, and $4 \%$ (34/512, 23/512, and 19/512 patients), respectively, for controls. The estimated pooled OR for the two trials showed significantly increased 14-month and 16-month SPF rates for patients who received T-DM1 compared with controls (OR, 1.85; 95\% CI, 1.19-2.87; $P=0.006$ and OR, 1.89; 95\% CI, $1.12-3.19 ; P=0.02$, respectively). However, the $18-$ month SPF rate was not significantly improved $(\mathrm{OR}, 1.53 ; 95 \% \mathrm{CI}$, $0.85-2.75 ; P=0.15)$. The overall Cochran's $Q$-test yielded a $P$-value of 0.68 , and the corresponding $I^{2}$ quantity was $0 \%$.

\section{Toxicity and adverse reactions}

The clinical trials included in this meta-analysis reported several AEs, including headache, ${ }^{19}$ pyrexia, ${ }^{19,21}$ epistaxis, ${ }^{19,21}$ constipation, ${ }^{19,21}$ cough, ${ }^{19,21}$ hypokalemia, ${ }^{19,21}$ pain in extremity, ${ }^{19,21}$ arthralgia, ${ }^{19}$ mucosal inflammation, ${ }^{25}$ dry mouth, ${ }^{21}$ alopecia, ${ }^{22}$ pneumonia, ${ }^{21}$ etc. Because some AEs occurred less frequently than others, we analyzed only the common AEs in this meta-analysis.

\section{Nonhematologic AEs}

Information on diarrhea, fatigue, and increased aspartate aminotransferase (AST) levels was available from three trials. $^{20,22,26}$ These three trials included a total of 1,700 patients (962 of whom received T-DM1 and 738 controls; Figure 6A). Compared with controls, fewer patients treated with T-DM1 experienced diarrhea (62\% versus $17 \%$ of patients), but more patients experienced fatigue (33\% versus 29\%) and increased AST levels ( $8 \%$ versus 18\%). Pooled analysis showed that diarrhea (OR, 0.19; 95\% CI, 0.06-0.61; $P=0.005$ ), fatigue
(OR, 1.29; 95\% CI, 1.04-1.59; $P=0.02$ ), and AST increases (OR, 3.33; 95\% CI, 1.43-7.76; $P=0.005)$ were significantly different between T-DM1-treated patients and controls (Figure 6A). Information on nausea, vomiting, and alanine aminotransferase (ALT) increases was available from two trials. ${ }^{20,22}$ These two trials included a total of 1,113 patients (559 of whom received T-DM1 treatment and 554 controls). Compared with control patients, nausea (45\% versus $40 \%$ ) and vomiting (29\% versus $20 \%$ ) occurred less frequently with T-DM1 treatment, whereas ALT increases occurred more frequently. Pooled analysis showed that vomiting (OR, 0.64; 95\% CI, 0.42-0.98; $P=0.04$ ) and ALT increases (OR, 2.90; 95\% CI, 1.20-7.01; $P=0.02$ ) were significantly different between the two groups, but nausea was not (OR, 0.89 ; 95\% CI, 0.61-1.28; $P=0.51$; Figure 6A). Information on dyspnea was available from two trials, ${ }^{20,26}$ which included a total of 722 patients (472 of whom received T-DM1). There was no significant difference in dyspnea between the T-DM1 and control groups (OR, 0.74; 95\% CI, 0.32-1.73; $P=0.49$; Figure 6A). Because there was a significant heterogeneity, a random-effects model was used.

\section{Hematologic AEs}

Information on neutropenia, anemia, and thrombocytopenia was available from three trials. ${ }^{20,22,26}$ These three trials included a total of 1,700 patients (962 of whom received T-DM1 and 738 controls). Compared with controls, T-DM1 treatment was associated with less neutropenia (17\% versus $6 \%$ of patients) but more thrombocytopenia ( $23 \%$ versus $3 \%$ ). Pooled analysis showed that the incidences of neutropenia (OR, 0.25; 95\% CI, 0.09-0.73; $P=0.01$ ) and thrombocytopenia (OR, 8.50; 95\% CI, 3.96-18.24; $P<0.00001)$ were significantly different between the two groups, but anemia was not (OR, $0.85 ; 95 \%$ CI, 0.45-1.61; $P=0.62$; Figure 6B). Information on febrile neutropenia and leukopenia was available from two trials, ${ }^{22,26}$ which included a total of 722 patients (472 of whom received T-DM1). Compared with controls, febrile neutropenia ( $6 \%$ versus $0 \%)$ and leukopenia $(11 \%$ versus $2 \%$ ) occurred less frequently with T-DM1 treatment. The pooled analysis showed that the incidences of febrile neutropenia (OR, 0.06; 95\% CI, 0.01-0.30; $P=0.0008)$ and leukopenia (OR, 0.22; 95\% CI, 0.08-0.57; $P=0.002$ ) were significantly different between patients treated with T-DM1 and controls (Figure 6B).

\section{Publication bias}

There was no evidence of publication bias for all outcomes through both Egger's test and Begg's test $(P>0.05)$. 


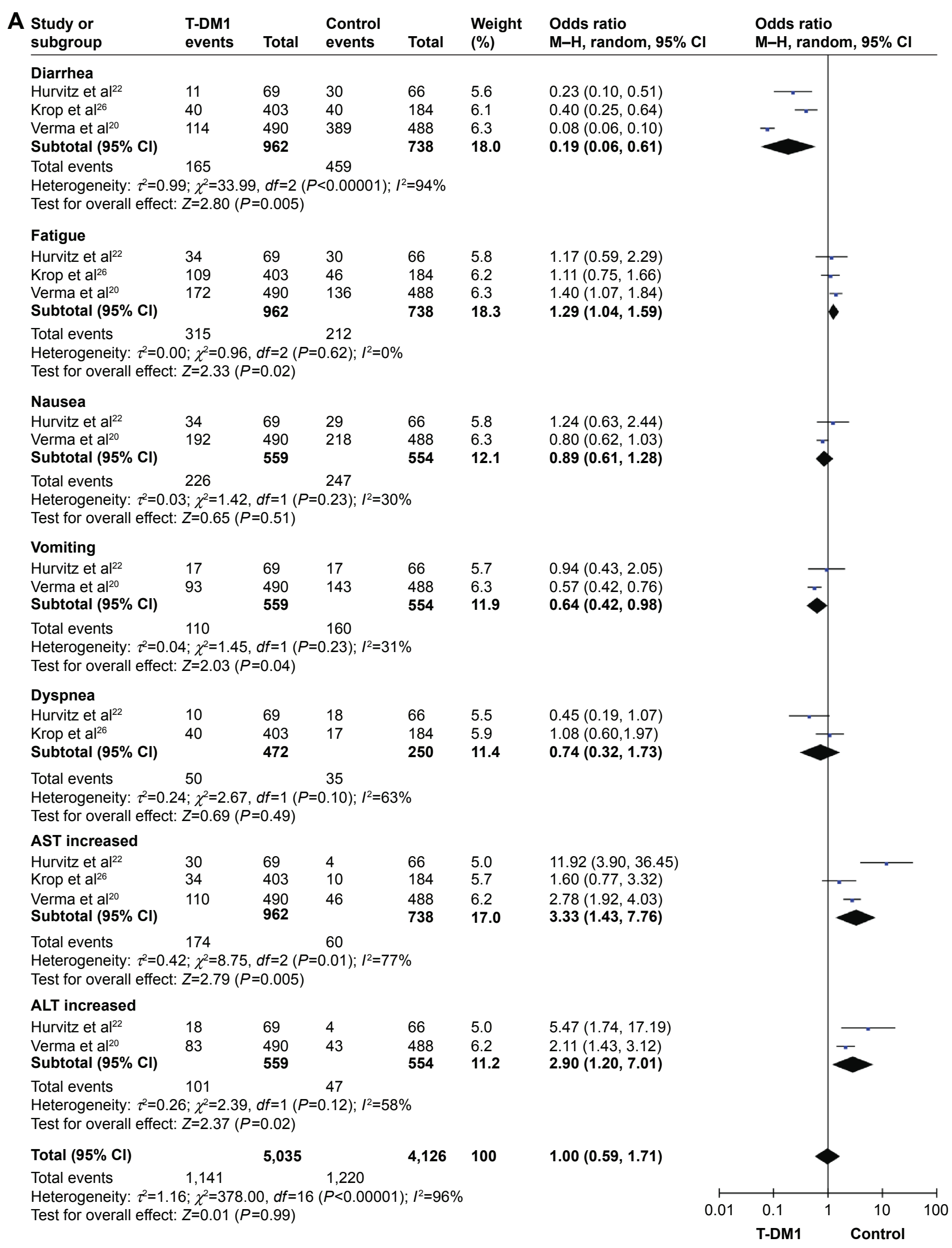

Figure 6 (Continued) 


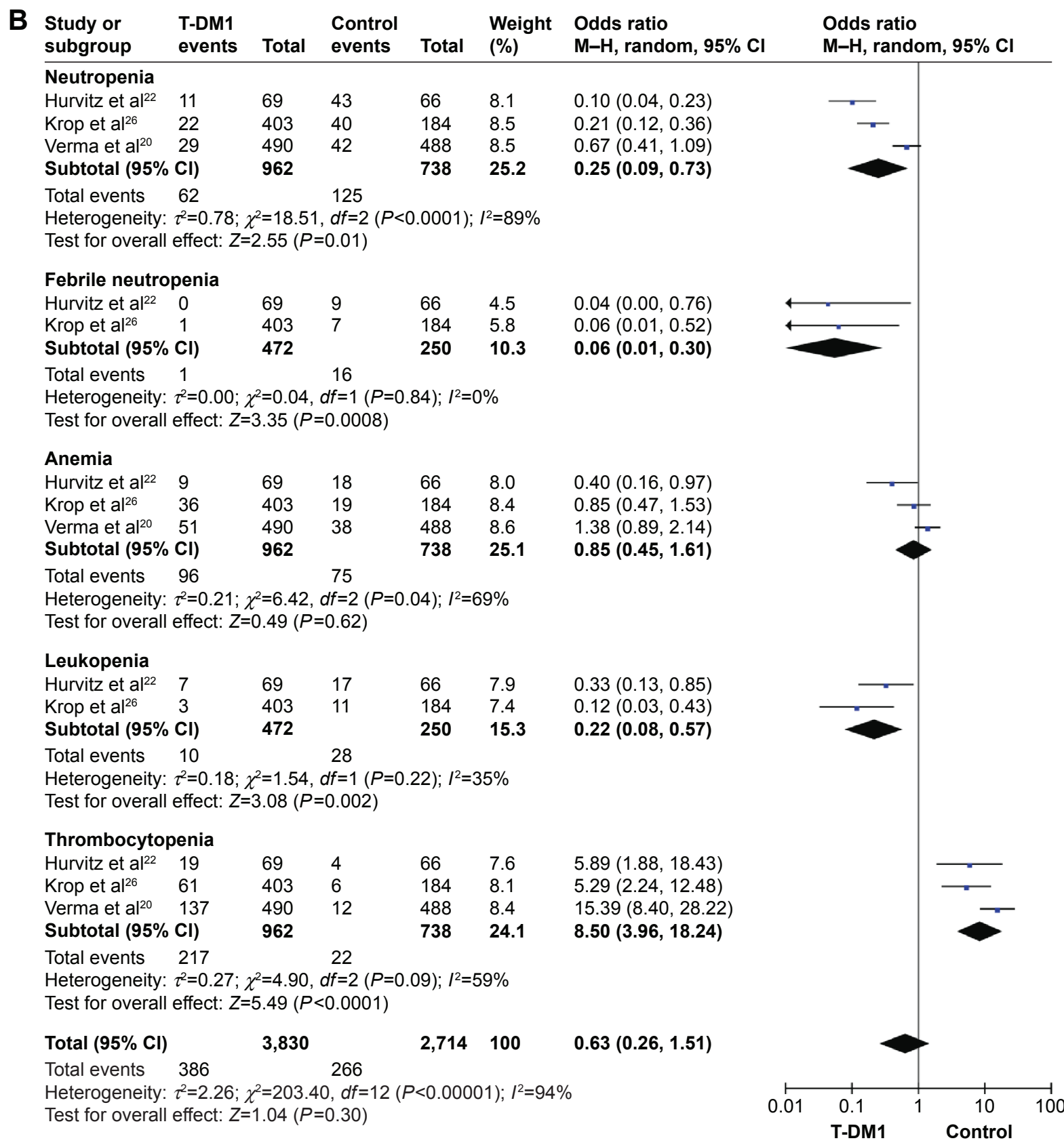

Figure 6 Forest plots of adverse event rates between patients undergoing T-DMI therapy and controls with respect to (A) nonhematologic and (B) hematologic adverse events.

Note: A random-effects meta-analysis model (M-H method) was used.

Abbreviations: AST, aspartate aminotransferase; ALT, alanine aminotransferase; Cl, confidence interval; df, degrees of freedom; M-H, Mantel-Haenszel; T-DMI, trastuzumab emtansine.

\section{Discussion}

Breast cancer is the most frequently diagnosed cancer and the leading cause of cancer death in women worldwide. ${ }^{27}$ HER2 overexpression is closely associated with a poor prognosis in some breast cancers. ${ }^{1}$ The effective treatment of HER2-overexpressing breast cancer after initial HER2directed therapy continues to represent an important medical need. ${ }^{19}$ A number of clinical trials have shown that HER2positive patients with breast cancer who receive T-DM1 have a more favorable prognosis than patients who do not receive T-DM1 or HER2-negative patients. T-DM1 was the first antibody-drug conjugate to use a thioether linker, which, in preclinical testing, proved to be more stable than other drugs used as linkers. ${ }^{28}$ In vitro studies showed that 
T-DM1-activated antibody-dependent cellular cytotoxicity inhibited HER2 receptor signaling and shedding of the HER2 extracellular domain in HER2-overexpressing cells from patients with breast cancer. ${ }^{29}$ In the current study, we performed a systemic analysis of the published clinical trials in order to assess the efficacy and safety of T-DM1 treatment in patients with breast cancer with respect to OS, PFS, ORR, SPF, and AEs.

Our analysis yielded several major findings. First, the overall meta-analysis showed that T-DM1 treatment could significantly improve the 2-month to 6-month, 8-month to 12-month, and 14-month to 16-month OS rates (all $P<0.0001$ ) of patients with breast cancer compared with controls Our comprehensive results calculated 2-month, 4-month, 6-month, 8-month, 10-month, 12-month, 14-month, and 16-month OS rates of $96 \%, 88 \%, 74 \%, 63 \%$, $54 \%, 42 \%, 33 \%$, and $27 \%$, respectively, which are slightly different to the results of independent trials; the median OS was $\sim 10$ months. Although the 10 -month OS rate was not significantly improved $(P=0.17)$, this may be explained because the number of patients included in this analysis was not large enough. Thus, the advantage of logistic regression is obvious. Our data analysis showed that T-DM1 treatment can significantly prolong OS in patients with breast cancer.

Second, the results also showed that T-DM1 treatment had a significant impact on 2-month to 6-month, 8-month to 12-month, and 14-month to 18-month PFS rates $(P<0.0001)$ compared with controls (Figure 3$)$. The summarized results showed that the 2-month, 4-month, 6-month, 8-month, 10-month, 12-month, 14-month, 16-month, and 18-month PFS rates were $83 \%, 65 \%, 42 \%, 31 \%, 21 \%$, $14 \%, 9 \%, 11 \%$, and $8 \%$, respectively, while the respective rates for controls were $76 \%, 56 \%, 33 \%, 24 \%, 14 \%, 9 \%$, $6 \%, 5 \%$, and $3 \%$, respectively. The median PFS time of patients treated with T-DM1 was between 4 months and 6 months. A previous study reported a median PFS time of 5.3 months for patients with breast cancer treated with T-DM1 (95\% CI, 3.6-8.9 months), ${ }^{19}$ while another Phase II clinical trial reported a median PFS of 5.5 months $(95 \% \mathrm{CI}$, 4.2-7.9 months). ${ }^{21}$ Although there were a few differences among the trials included in this meta-analysis, the positive trend was fully confirmed. However, T-DM1 therapy was not found to significantly extend the 4-month PFS rate compared with controls $(P=0.06)$. This may be because the number of patients included in this analysis was not large enough. Through logistic regression, however, our analysis revealed that T-DM1 therapy has a significant influence on PFS.
Furthermore, the secondary endpoints of ORR and SPF showed favorable results in the T-DM1 treatment group compared with corresponding controls ( $P \leq 0.0001$; Figures 4 and 5). Our pooled analysis of the collected data showed that the 2-month, 4-month, 6-month, 8-month, 10-month, 12-month, 14-month, 16-month, and 18-month SPF rates were $77 \%, 57 \%, 37 \%, 29 \%, 20 \%, 15 \%, 12 \%, 8 \%$, and $6 \%$, respectively, compared with $67 \%, 45 \%, 37 \%, 19 \%$, $12 \%, 10 \%, 7 \%, 4 \%$, and $4 \%$, respectively, for controls. The 2-month, 10-month, and 18-month SPF rates were not significantly improved versus controls $(P=0.06, P=0.08$, and $P=0.15$, respectively). We should note that the SPF analysis included only two trials with a total of 1,027 patients for each endpoint; a larger number of trials are required to prove these results.

The analysis also showed that nonhematologic and hematologic AEs were not significantly different between the T-DM1 and control groups ( $P=0.99$ and $P=0.30$, respectively; Figure 6). Overall, therefore, according to the present study, T-DM1 treatment may prove advantageous for HER2positive patients with breast cancer.

There are some points that may explain these results. First, T-DM1 has shown antitumor activity against breast cancer tumors and HER2-positive cancer cell lines that do not respond or that have developed resistance to trastuzumab or lapatinib. ${ }^{15,28,29}$ Furthermore, T-DM1 was the first HER2targeted agent to demonstrate a significant clinical activity in patients with breast cancer who had progressed on both lapatinib- and trastuzumab-based regimens. ${ }^{13}$ Second, T-DM1 has been shown to inhibit PI3K in cells that are insensitive to trastuzumab. ${ }^{30,31}$ In addition, T-DM1 has been seen to inhibit the growth of breast cancer cells that are resistant to lapatinib and have an activated PI3K pathway. ${ }^{29,32-35}$ Third, the maximal cytotoxicities in antibody-dependent cell-mediated cytotoxicity assay have been reported to be $57 \%$ with T-DM1 and 48\% trastuzumab. ${ }^{10,36,37}$ In addition, the active metabolite of T-DM1, lysine-Ne-4-( $N$-maleimidomethyl) cyclohexane-1-carboxylate-DM1 is released when T-DM1 is internalized. Because lysine-Ne-( $N$-maleimidomethyl) cyclohexane-1-carboxylate-DM1 is a zwitterion, it does not readily cross the plasma membrane of neighboring normal cells. This likely contributes to the overall safety profile of T-DM1. ${ }^{33,38,39}$ Finally, Yu et $\mathrm{al}^{27}$ have concluded that the regimen of T-DM1 as well as pertuzumab in combination with trastuzumab and docetaxel is efficacious with fewer side effects as compared with other regimens through a network meta-analysis of six HER2-targeted treatment drugs and one naive standard treatment. Although that article only included 
a clinical study of T-DM1, it has been shown that T-DM1 is a better regimen.

In short, T-DM1 therapy for patients with breast cancer associated with significantly prolonged OS and PFS improved ORR and SPF and low rates of AEs.

\section{Limitations}

The reliability of this meta-analysis might be influenced by several factors. First, not all the trials included in this systemic review were multicenter clinical trials, so the results of the present meta-analysis cannot be extended to all patients with breast cancer across the world. Second, some clinical trials with good efficacy were excluded because they lacked appropriate control arms; thus, the validity of the study results might be underestimated. Third, not all the eight clinical trials included in this study were randomized controlled trials, and only two trials were Phase III trials with large samples. Therefore, this review might contain distribution and implementation biases. Finally, we included data on patients with breast cancer from published articles, rather than drawing the first-hand data from patient records. In addition, negative trial outcomes often remain unpublished. Thus, our analysis might have resulted in an overestimation of the effects of T-DM1 treatment. Finally, the results of our study may be misleading because of the different design of clinical trials included in this meta-analysis. These factors might also introduce bias into the conclusions. However, we believe that this study is valuable in improving the design of randomized controlled multicenter clinical trials.

\section{Future perspectives}

In the near future, T-DM1 will be widely used in the treatment of HER2-positive patients with breast cancer, and the costs of the therapy will be reduced. But before that, there are still some unanswered questions about T-DM1 which need to be solved. First of all, for which kinds of patients is T-DM1 most effective? We urgently need to identify biomarkers that will help to identify those patients who are most likely to benefit from T-DM1. ${ }^{8,40}$ In addition, we still need to definitively address the mechanisms of resistance to T-DM1; such research will require tumor tissue from patients with progressive disease, and biopsies from these patients should be included in future trials of T-DM1.,41,42 Furthermore, we also need to summarize and explore the best optimal dose and dosing method for T-DM1. Finally, with the continuous progress that is being made in biotechnology, the future treatment of patients with breast cancer will move toward individualized therapy.

\section{Conclusion}

Overall, this meta-analysis of T-DM1 in HER2-positive patients with breast cancer has yielded encouraging results with superiority in OS and PFS, improvements in ORR and SPF, and low rates of AEs. Hence, these results suggest that T-DM1 has a great potential as an efficacious clinical therapy for the treatment of HER2-positive patients with breast cancer who were previously treated with trastuzumab, lapatinib, or other standard-directed therapies.

\section{Acknowledgment}

This research work was supported by the Natural Science Foundation of Zhejiang Province (No LQ12H16002) and Science and Technology Plan Projects of Huzhou (No 2013GZ13).

\section{Disclosure}

The authors report no conflicts of interest in this work.

\section{References}

1. Boyraz B, Sendur MA, Aksoy S, et al. Trastuzumab emtansine (T-DM1) for HER2-positive breast cancer. Curr Med Res Opin. 2013;29(4): 405-414.

2. Barok M, Joensuu H, Isola J. Trastuzumab emtansine: mechanisms of action and drug resistance. Breast Cancer Res. 2014;16(2):209.

3. Figueroa-Magalhães MC, Jelovac D, Connolly RM, Wolff AC. Treatment of HER2-positive breast cancer. Breast. 2014;23(2): 128-136.

4. Girish S, Gupta M, Wang B, et al. Clinical pharmacology of trastuzumab emtansine (T-DM1): an antibody-drug conjugate in development for the treatment of HER2-positive cancer. Cancer Chemother Pharmacol. 2012;69(5):1229-1240.

5. Krishnamurti U, Silverman JF. HER2 in breast cancer: a review and update. Adv Anat Pathol. 2014;21(2):100-107.

6. Incorvati JA, Shah S, Mu Y, Lu J. Targeted therapy for HER2 positive breast cancer. J Hematol Oncol. 2013;6:38.

7. LoRusso PM, Weiss D, Guardino E, Girish S, Sliwkowski MX. Trastuzumab emtansine: a unique antibody-drug conjugate in development for human epidermal growth factor receptor 2-positive cancer. Clin Cancer Res. 2011;17(20):6437-6447.

8. Krop I, Winer EP. Trastuzumab emtansine: a novel antibody-drug conjugate for HER2-positive breast cancer. Clin Cancer Res. 2014 20(1):15-20.

9. Dawood S, Broglio K, Buzdar AU, Hortobagyi GN, Giordano SH. Prognosis of women with metastatic breast cancer by HER2 status and trastuzumab treatment: an institutional-based review. J Clin Oncol. 2010; 28(1):92-98.

10. Ballantyne A, Dhillon S. Trastuzumab emtansine: first global approval. Drugs. 2013;73(7):755-765.

11. Diessner J, Bruttel V, Stein RG, et al. Targeting of preexisting and induced breast cancer stem cells with trastuzumab and trastuzumab emtansine (T-DM1). Cell Death Dis. 2014;5:e1149.

12. Singh JC, Jhaveri K, Esteva FJ. HER2-positive advanced breast cancer: optimizing patient outcomes and opportunities for drug development Br J Cancer. 2014;111(10):1888-1898.

13. Phillips GD, Fields CT, Li G, et al. Dual targeting of HER2-positive cancer with trastuzumab emtansine and pertuzumab: critical role for neuregulin blockade in antitumor response to combination therapy. Clin Cancer Res. 2014;20(2):456-468. 
14. Yamamoto-Ibusuki M, Arnedos M, André F. Targeted therapies for ER+/HER2- metastatic breast cancer. BMC Med. 2015;13:137.

15. Lewis Phillips GD, Li G, Dugger DL, et al. Targeting HER2-positive breast cancer with trastuzumab-DM1, an antibody-cytotoxic drug conjugate. Cancer Res. 2008;68:9280-9290.

16. Barok M, Tanner M, Köninki K, Isola J. Trastuzumab-DM1 causes tumour growth inhibition by mitotic catastrophe in trastuzumab-resistant breast cancer cells in vivo. Breast Cancer Res. 2011;13(2):R46.

17. Austin CD, Wen X, Gazzard L, Nelson C, Scheller RH, Scales SJ. Oxidizing potential of endosomes and lysosomes limits intracellular cleavage of disulfide-based antibody-drug conjugates. Proc Natl Acad Sci U S A. 2005;102(50):17987-17992.

18. DerSimonian R, Laird N. Meta-analysis in clinical trials. Control Clin Trials. 1986;7(3):177-188.

19. Burris HA 3rd, Rugo HS, Vukelja SJ, et al. Phase II study of the antibody drug conjugate trastuzumab-DM1 for the treatment of human epidermal growth factor receptor 2 (HER2)-positive breast cancer after prior HER2-directed therapy. J Clin Oncol. 2011;29(4):398-405.

20. Verma S, MilesD, Gianni L, etal. Trastuzumabemtansine for HER2-positive advanced breast cancer. $N$ Engl J Med. 2012;367(19):1783-1791.

21. Krop IE, LoRusso P, Miller KD, et al. A phase II study of trastuzumab emtansine in patients with human epidermal growth factor receptor 2-positive metastatic breast cancer who were previously treated with trastuzumab, lapatinib, an anthracycline, a taxane, and capecitabine. J Clin Oncol. 2012;30(26):3234-3241.

22. Hurvitz SA, Dirix L, Kocsis J, et al. Phase II randomized study of trastuzumab emtansine versus trastuzumab plus docetaxel in patients with human epidermal growth factor receptor 2-positive metastatic breast cancer. J Clin Oncol. 2013;31(9):1157-1163.

23. Welslau M, Diéras V, Sohn JH, et al. Patient-reported outcomes from EMILIA, a randomized phase 3 study of trastuzumab emtansine (T-DM1) versus capecitabine and lapatinib in human epidermal growth factor receptor 2-positive locally advanced or metastatic breast cancer. Cancer. 2014;120(5):642-651.

24. Perez EA, Hurvitz SA, Amler LC, et al. Relationship between HER2 expression and efficacy with first-line trastuzumab emtansine compared with trastuzumab plus docetaxel in TDM4450g: a randomized phase II study of patients with previously untreated HER2-positive metastatic breast cancer. Breast Cancer Res. 2014;16(3):R50.

25. Miller KD, Diéras V, Harbeck N, et al. Phase IIa trial of trastuzumab emtansine with pertuzumab for patients with human epidermal growth factor receptor 2-positive, locally advanced, or metastatic breast cancer. J Clin Oncol. 2014;32(14):1437-1444.

26. Krop IE, Kim SB, González-Martín A, et al. Trastuzumab emtansine versus treatment of physician's choice for pretreated HER2-positive advanced breast cancer (TH3RESA): a randomised, open-label, phase 3 trial. Lancet Oncol. 2014;15(7):689-699.

27. Yu Q, Zhu Z, Liu Y, Zhang J, Li K. Efficacy and safety of HER2-targeted agents for breast cancer with HER2-overexpression: a network metaanalysis. PLoS One. 2015;10(5):e0127404.

28. Michel LL, Bermejo JL, Gondos A, Marmé F, Schneeweiss A. T-DM1 as a new treatment option for patients with metastatic HER2-positive breast cancer in clinical practice. Anticancer Res. 2015;35(9):5085-5090.
29. Junttila TT, Li G, Parsons K, Phillips GL, Sliwkowski MX. Trastuzumab-DM1 (T-DM1) retains all the mechanisms of action of trastuzumab and efficiently inhibits growth of lapatinib insensitive breast cancer. Breast Cancer Res Treat. 2011;128(2):347-356.

30. Erickson HK, Park PU, Widdison WC, et al. Antibody-maytansinoid conjugates are activated in targeted cancer cells by lysosomal degradation and linker-dependent intracellular processing. Cancer Res. 2006; 66(8):4426-4433.

31. Saad OM, Shen BQ, Xu K, Khojasteh SC, Girish S, Kaur S. Bioanalytical approaches for characterizing catabolism of antibody-drug conjugates. Bioanalysis. 2015;7(13):1583-1604.

32. Chung YC, Kuo JF, Wei WC, Chang KJ, Chao WT. Caveolin-1 dependent endocytosis enhances the chemosensitivity of HER-2 positive breast cancer cells to trastuzumab emtansine (T-DM1). PLoS One. 2015;10(7):e0133072.

33. Junutula JR, Flagella KM, Graham RA, etal. Engineered thio-trastuzumabDM1 conjugate with an improved therapeutic index to target human epidermal growth factor receptor 2-positive breast cancer. Clin Cancer Res. 2010;16(19):4769-4778.

34. Piwko C, Prady C, Yunger S, Pollex E, Moser A. Safety profile and costs of related adverse events of trastuzumab emtansine for the treatment of HER2-positive locally advanced or metastatic breast cancer compared to capecitabine plus lapatinib from the perspective of the Canadian health-care system. Clin Drug Investig. 2015;35(8):487-493.

35. Shen $\mathrm{BQ}$, Bumbaca D, Yue Q, et al. Non-clinical disposition and metabolism of DM1, a component of trastuzumab emtansine (T-DM1), in Sprague Dawley Rats. Drug Metab Lett. 2015;9(2):119-131.

36. Miranda Romero P, Marín Gil R. Trastuzumab emtansine in locally advanced or metastatic HER2 positive breast cancer; GENESIS-SEFH drug evaluation report. Farm Hosp. 2015;39(3):171-175.

37. Esteva FJ, Miller KD, Teicher BA. What can we learn about antibodydrug conjugates from the T-DM1 experience? Am Soc Clin Oncol Educ Book. 2015;35:e117-e125.

38. Van den Mooter T, Teuwen LA, Rutten A, Dirix L. Trastuzumab emtansine in advanced human epidermal growth factor receptor 2-positive breast cancer. Expert Opin Biol Ther. 2015;15(5):749-760.

39. Peddi PF, Hurvitz SA. Ado-trastuzumab emtansine (T-DM1) in human epidermal growth factor receptor 2 (HER2)-positive metastatic breast cancer: latest evidence and clinical potential. Ther Adv Med Oncol. 2014; 6(5):202-209.

40. Torres S, Maralani P, Verma S. Activity of T-DM1 in HER-2 positive central nervous system breast cancer metastases. BMJ Case Rep. 2014.

41. Yu L, Wang Y, Yao Y, et al. Eradication of growth of HER2-positive ovarian cancer with trastuzumab-DM1, an antibody-cytotoxic drug conjugate in mouse xenograft model. Int $J$ Gynecol Cancer. 2014; 24(7):1158-1164.

42. Giordano SH, Temin S, Kirshner JJ, et al; American Society of Clinical Oncology. Systemic therapy for patients with advanced human epidermal growth factor receptor 2-positive breast cancer: American Society of Clinical Oncology clinical practice guideline. J Clin Oncol. 2014; 32(19):2078-2099.
OncoTargets and Therapy

\section{Publish your work in this journal}

OncoTargets and Therapy is an international, peer-reviewed, open access journal focusing on the pathological basis of all cancers, potential targets for therapy and treatment protocols employed to improve the management of cancer patients. The journal also focuses on the impact of management programs and new therapeutic agents and protocols on
Dovepress

patient perspectives such as quality of life, adherence and satisfaction. The manuscript management system is completely online and includes a very quick and fair peer-review system, which is all easy to use. Visit http://www.dovepress.com/testimonials.php to read real quotes from published authors. 